\title{
Intoxication by Hand-Sanitizers and other Toxic Alcohols in a Low-Resource Setting: Two Case Reports
}

\author{
Micah LA Heldeweg ${ }^{1}$, Louisa G Kluijver² ${ }^{2}$ Kenrick Berend ${ }^{3}$ \\ ${ }^{1}$ Department of Intensive Care, Amsterdam University Medical Centers, location VUmc, Amsterdam, the Netherlands \\ ${ }^{2}$ Department of Internal Medicine, Alrijne Hospital, Leiden, the Netherlands \\ ${ }^{3}$ Department of Internal Medicine, Curaçao Medical Center, Curaçao
}

Received: 28/06/2021

Accepted: $12 / 07 / 2021$

Published: 03/08/2021

\begin{abstract}
How to cite this article: Heldeweg MLA, Kluijver LG, Berend K. Intoxication by hand-sanitizers and other toxic alcohols in a low-resource setting: two case
\end{abstract} reports. EJCRIM 2021;8: doi:10.12890/2021_002751.

Conflicts of Interests: The authors declare there are no competing interests.

Disclosures: Data from case 2 have been previously published in a commercial journal (Limper M, Berend K. Int J Intensive Care 2010: p99-101). The data have been reproduced with permission from the previous authors.

This article is licensed under a Commons Attribution Non-Commercial 4.0 License

\section{ABSTRACT}

Toxic alcohol poisoning can be lethal if not identified early and treated appropriately. Toxic alcohol assays are often unavailable in lowresource setting, so clinicians have to infer a diagnosis based on suspicion, repeated evaluation and biochemical course. We report a case of toxic alcohol poisoning concealed by auto-intoxication with in-hospital hand sanitizer. The eventual appearance of a concurrent high anion gap prompted dialysis. In another case, a comatose patient presented with a high osmolal gap and a high anion gap. Incorrect a priori opinions caused us to defer dialysis and the patient died shortly afterwards. Clinicians should be aware that toxic alcohol poisoning can produce a confusing diagnostic picture with an insidious course, and that doctor delay can prove fatal.

\section{LEARNING POINTS}

- Toxic alcohol ingestion may be lethal and warrants early identification, but this is not always possible.

- Incorrect a priori opinions by clinicians, or the co-ingestion of other alcohols by a patient, may produce a confusing diagnostic picture.

- Physicians should not defer immediate treatment for patients suspected of toxic alcohol ingestion with a double gap or visual disturbances.

\section{KEYWORDS}

Toxic alcohols, anion gap, osmolal gap, doctor delay

\section{INTRODUCTION}

Toxic alcohol poisoning can be deadly, but timely identification may be challenging, requiring a high level of suspicion. This case report discusses two cases which highlight the fact that even when patients are (biochemically) intensively monitored, the course of an intoxication can be insidious and fatal.

\section{CASE DESCRIPTIONS}

Case 1, the recurring osmolal gap

A 57-year-old man with an unknown medical history presented to the emergency department with abdominal cramps, vomiting and strange behaviour. He had been found in his cabin on a cargo ship with a half-empty $100 \mathrm{ml}$ bottle of hand-sanitizer (ethanol 62\%) and another empty $100 \mathrm{ml}$ bottle of hand-sanitizer (isopropanol 10\%). An accompanying ship employee disclosed that the patient was an avid drinker, had vomited three times following ingestion, and was brought to the emergency department 3 hours later. 
On examination, the patient was agitated, sweaty and tremulous. His temperature was $36.8^{\circ} \mathrm{C}$, blood pressure $126 / 87 \mathrm{mmHg}$, pulse 93 beats per minute, respiratory rate 20 breaths per minute, and oxygen saturation $94 \%$ on ambient air. There was mild pain upon palpation in the epigastric region. The rest of the examination revealed no other abnormalities.

Laboratory values at admission showed high anion gap metabolic acidosis (HAGMA), metabolic alkalosis and an osmolal gap (OG) of 61 with moderately elevated lactate (Table 1). Urine analysis showed no glycosuria, ketonuria or calcium oxalate crystals. Other haematological and biochemical parameters, including amylase, were normal.

\begin{tabular}{|l|l|l|l|}
\hline \multicolumn{1}{|c|}{ Test } & \multicolumn{1}{|c|}{ Case 1 } & \multicolumn{1}{c|}{ Case 2 } & \multicolumn{1}{c|}{ Reference range } \\
\hline$p H$ & 7.47 & 7.22 & $7.35-7.45$ \\
\hline$p C O 2$ & 4.4 & 2.8 & $4.7-6.0 \mathrm{kPa}$ \\
\hline$p O 2$ & 12.0 & 17.6 & $9.3-13.3 \mathrm{kPa}$ \\
\hline HCO3 & 23.7 & 8.2 & $22-26 \mathrm{mmol} / \mathrm{l}$ \\
\hline O2 saturation & $94 \%$ & $98 \%$ & $95-100 \%$ \\
\hline Haemoglobin & 15.0 & 13.2 & $12.0-16.0 \mathrm{~g} / \mathrm{dl}$ \\
\hline Leucocytes & 11.7 & 23.9 & $3.5-10.5 \times 109 / \mathrm{l}$ \\
\hline CRP & 1.8 & $<5$ & $<8 \mathrm{mg} / \mathrm{l}$ \\
\hline Sodium & 136 & 136 & $135-145 \mathrm{mmol} / \mathrm{l}$ \\
\hline Potassium & 3.5 & 2.4 & $3.5-5.0 \mathrm{mmol} / \mathrm{l}$ \\
\hline Chloride & 95 & 102 & $98-107 \mathrm{mmol} / \mathrm{l}$ \\
\hline Creatinine & 41 & 143 & $70-110 \mu \mathrm{mol} / \mathrm{l}$ \\
\hline
\end{tabular}

The patient was admitted to hospital with poisoning by toxic alcohol(s). He was treated with fluid infusion, benzodiazepines, thiamine, pyridoxine and a proton-pump inhibitor ${ }^{[1]}$. The criteria for starting ethanol infusion, 4-methylpyrazole infusion (not available in our hospital) or haemodialysis include either documented or laboratory proven poisoning, or clinical suspicion with at least two of the following criteria: arterial $\mathrm{pH}<7.3$, serum bicarbonate $<20 \mathrm{mmol} / \mathrm{I}$ or $\mathrm{OG}>10 \mathrm{mOsm} /{ }^{[2]}$. Since only one of the criteria was fulfilled, ethanol infusion and dialysis were withheld.

During admission, recurrent episodes of rising OG and HAGMA occurred even though the patient was restrained for agitation (Fig. 1). However, the only options for recurrent intoxication were the bottles of hand sanitizer containing ethanol $62 \%$ and isopropanol $10 \%$ attached to the ends of all beds on the ward (Fig. 2). The hand sanitizer at the patient's bed was filled with water and within half an hour, he had reached for it and attempted to drink its contents, confirming our suspicion of auto-intoxication (Fig. 3).

At night, the patient went to the pantry and drank half a bottle of $250 \mathrm{ml}$ hand sanitizer, once again leading to HAGMA, OG and ketonuria. The unexpected reappearance of a double gap prompted us to perform haemodialysis, lowering the OG to zero the same day. The patient was discharged soon afterwards.

\section{DISCUSSION}

Our hospital does not have access to timely biochemical alcohol toxicology determination. Thus, we have to infer a diagnosis based on history and biochemical values.

Considering a $90 \mathrm{~kg}$ patient with a distribution volume of 54 litres $(0.6 \mathrm{l} / \mathrm{kg})$ and a conservative estimation of ethanol elimination rate (15 $\mathrm{mg} / 100 \mathrm{ml} /$ hour ), we can calculate blood ethanol content using a simplified linear method based on the Widmark formula (Fig. 4) ${ }^{[3,4]}$. 
Internal Medicine

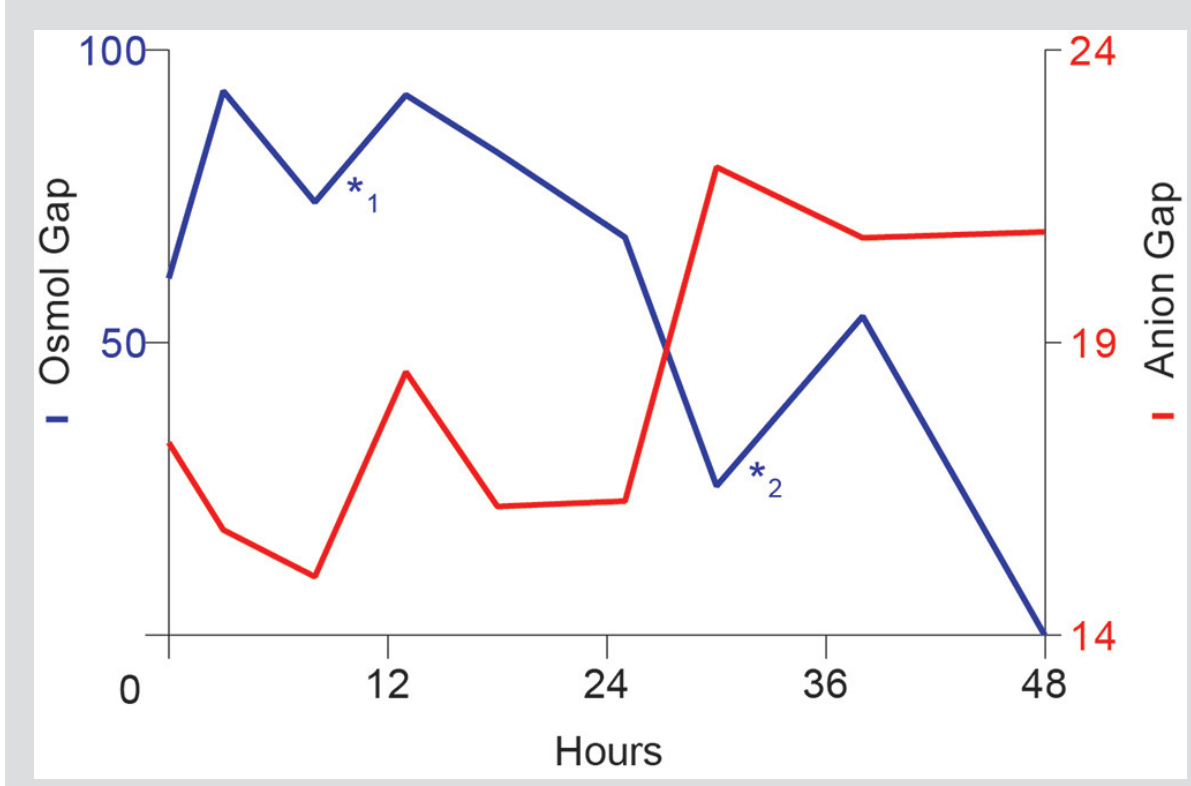

Figure 1. Time course of osmolal gap and anion gap during admission. ${ }^{*} 1$ and ${ }^{*} 2$ indicate the moment of hand sanitizer autointoxication
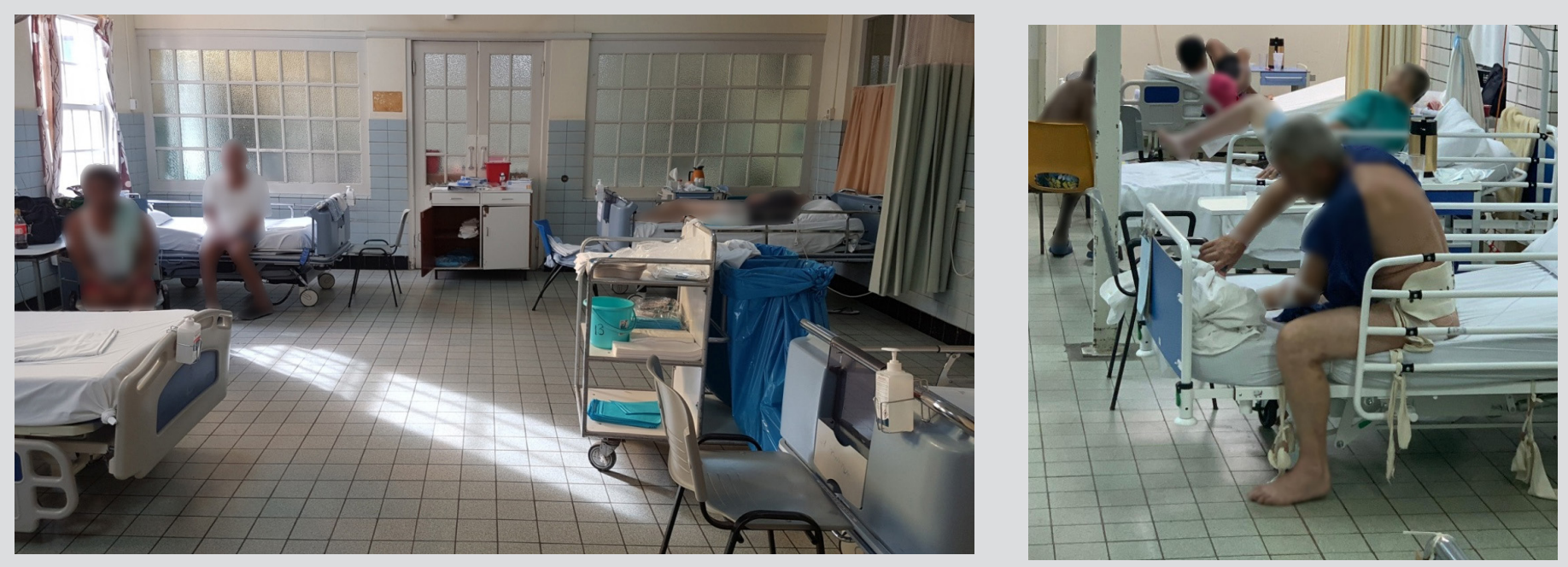

Figure 2. Hand sanitizers attached to the ends of patients' beds

Figure 3. The patient attempting to drink the fake hand sanitize

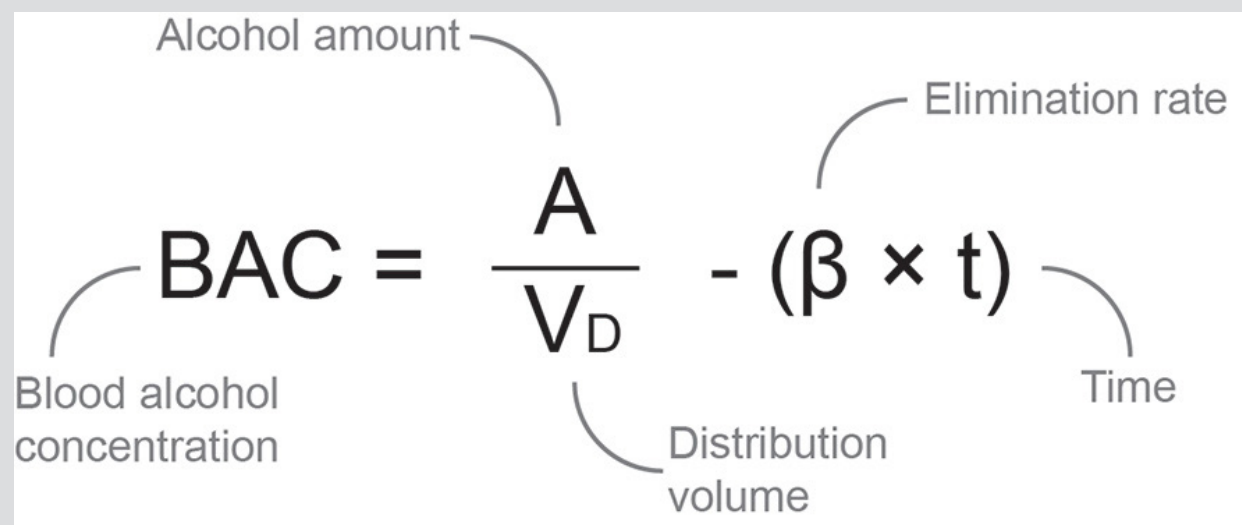

Figure 4. Time course of osmolal gap and anion gap during admission 
For isopropanol we did the same calculation, but omitted an elimination rate because of the co-presence of ethanol, retaining a liberal estimation of peak blood alcohol content at the time of presentation. The calculated blood ethanol and isopropanol concentrations were 93.1 and $21.3 \mathrm{mg} / \mathrm{dl}$, respectively, corresponding to a rise in osmolality of $23.3 \mathrm{mOsm} / \mathrm{l}$. Even with this liberal estimation, the prehospital hand-sanitizer ingestion did not appear to explain the OG of 63, let alone its rise to $93 \mathrm{mOsmol} / \mathrm{kg}$.

Moreover, the patient also had HAGMA. Although marginal acidosis can also occur in acute ethanol ingestion, the presence of an overt double gap suggests co-intoxication (Table 2) ${ }^{[5,6]}$. Toxic alcohols are metabolized to acidic compounds through alcohol dehydrogenase $(A D H)$, simultaneous decreasing $O G$ and increasing HAGMA ${ }^{[7]}$. Exceptions are isopropanol and propylene glycol, which do not form acidic metabolites. Considering alcohols, ethanol has the greatest affinity for ADH and at a serum concentration of $100 \mathrm{mg} / \mathrm{dl}$, it completely saturates $\mathrm{ADH}$. As a result, ethanol can inhibit the formation of metabolites and delay symptoms ${ }^{[8,9]}$.

\begin{tabular}{|c|c|c|c|}
\hline Substance & Symptoms & $\begin{array}{l}\Delta \text { Serum osmolality per } \\
10 \mathrm{mg} / \mathrm{dl} \text { increase in } \\
\text { serum concentration }\end{array}$ & $\begin{array}{l}\text { Acidosis onset } \\
\text { (with ethanol } \\
\text { ingestion) }\end{array}$ \\
\hline Ethanol & $\begin{array}{l}\text { Slurred speech, disinhibition, incoordination, nystagmus, stupor, pancreatitis, } \\
\text { coma }\end{array}$ & 2.12 & No \\
\hline Diethylene glycol & $\begin{array}{l}\text { Abdominal pain, diarrhoea, drunkenness, renal failure, acidosis, pancreatitis, } \\
\text { hepatitis, seizure, cranial and peripheral neuropathy; facial diplegia (50\%) }\end{array}$ & 0.9 & $\begin{array}{l}\text { Yes, after } 24 \text { hours } \\
\text { (48 hours) }\end{array}$ \\
\hline Ethylene glycol & $\begin{array}{l}\text { Nausea, vomiting, approximately } 86 \% \text { of patients will be acidotic at presentation } \\
\text { After } 4-12 \mathrm{~h} \text { : neurological symptoms (CNS depression, hyporeflexia, convulsions) } \\
\text { After } 24-72 \mathrm{~h} \text { : renal failure }\end{array}$ & 1.60 & $\begin{array}{l}\text { Yes, after } 12 \text { hours } \\
\text { (48 hours) }\end{array}$ \\
\hline Isopropanol & $\begin{array}{l}\text { Rapid intoxication within 30-60 min. Drunk appearance, somnolence, coma, } \\
\text { respiratory depression, hypotension, fruity acetone fetor }\end{array}$ & 1.66 & No \\
\hline Propylene glycol & $\begin{array}{l}\text { Agitation, haemolysis, arrhythmia, renal failure } \\
\text { High risk: children, patients with liver or renal failure, burns after application } \\
\text { on the skin }\end{array}$ & 1.31 & $\begin{array}{l}\text { No, only in presence } \\
\text { of lactic acidosis }\end{array}$ \\
\hline
\end{tabular}

Table 2. Differential diagnosis of a high anion gap/high osmolal gap metabolic acidosis

Because he worked on a cargo ship, we suspected possible access to industrial fluids or homebrew alcohol containing methanol, ethylene glycol or diethylene glycol ${ }^{[5,7]}$. The absence of visual or neurological deficits did not support methanol intoxication. Lack of urine calcium oxalate crystals was an argument against ethylene glycol. Diethylene glycol ingestion is often accompanied by elevated amylase, liver enzymes and creatinine, while having the lowest osmotic concentration, making it less likely in our patient. With none of the dialysis criteria fulfilled and no clinical support to identify specific toxic alcohols, we chose a watchful waiting strategy initially.

The episodes of recurrent increased OG were surprising. The presence of competing alcohols can impede metabolism, but not reverse it. Therefore, the recurrent rise in OG during admission was caused by renewed auto-intoxication. Mortality in ethanol and isopropanol intoxication is low, but the striking wide double gap prompted dialysis treatment ${ }^{[5]}$. Repeated auto-intoxications with ethanol might have kept the metabolites of another concealed toxic alcohol poisoning at bay by saturating ADH activity ${ }^{[7]}$. Although we were not able to identify the culprit toxic alcohol prior to dialysis, we did not wish to risk a fatal doctor delay.

\section{Case 2, fatal doctor delay}

A 49-year-old woman without a relevant medical history was found unresponsive by her children who called the emergency number. Upon arrival, ambulance staff initiated cardiopulmonary resuscitation and performed intubation. Spontaneous circulation returned within 15 minutes. The patient was transferred to our hospital. According to family members, the patient reported vertigo, nausea and vomiting. Her general practitioner had treated her with clarithromycin for suspected sinusitis 1 day before admission. The patient was divorced and living with her two children. She did not smoke, drink alcohol or use illicit drugs. According to her family, friends and the general practitioner, she was not depressed or suicidal. No empty bottles, pill strips or other traces of auto-intoxication were found in her home. 
On examination, the patient was in a comatose state (E1M1Vtube) with normal pupillary light reflexes. No other neurological symptoms were observed. Temperature was $35.6^{\circ} \mathrm{C}$, blood pressure $110 / 60 \mathrm{mmHg}$, and pulse 130 beats per minute. The remaining examination revealed no abnormalities.

Computed tomography of the brain was normal. At lumbar puncture, opening pressure and laboratory fluid analysis were normal.

Laboratory values indicated HAGMA and a high OG, with an increased lactate level (Table 1). Urine analysis showed trace ketones and an 'amorphous residue' but no calcium oxalate crystals.

The patient was transferred to the intensive care unit where she developed refractory hypotension. Haemodialysis was considered but not started; instead, the patient received supportive care and died 45 hours after admission. Post-mortem serum analysis, performed in another hospital, showed a very high concentration of ethylene glycol (>2 ml/kg), revealing the cause of death.

\section{DISCUSSION}

Toxic alcohol poisoning was an important possibility in this patient considering her sudden comatose state without signs of infection, a cerebrovascular event or bleeding. Clinical suspicion was raised due to lactate acidosis and a high OG without ketoacidosis or uraemia [10,11] Despite the OG of $20 \mathrm{mOsm} / \mathrm{l}$ at presentation, we did not pursue the diagnosis of toxic alcohol poisoning for several reasons. We believed that there was a very low a priori probability of such poisoning. The patient had two children who had been with her that morning, and they were unaware of any strange behaviour or alcohol use. No empty bottles of toxic alcohols or pill strips were found in the house. Therefore, alcohol intoxication seemed unlikely.

We evaluated the differential diagnosis of combined HAGMA and OG (Table 2). The OG was considered relatively low for a recent methanol intoxication ${ }^{[12]}$. Absence of calcium oxalate crystals in urine was an argument against ethylene glycol. Diethylene glycol was considered unlikely due to its low osmotic concentration and extremely rare occurrence.

The OG may be helpful in detecting probable toxic alcohol poisoning, but its limitations in both sensitivity and specificity must be considered ${ }^{[7]}$. Haemodialysis was not performed due to hypotension and assumed insufficient evidence to start this therapy. Retrospectively, ethanol infusion could have been started while final toxicological results were awaited. The source of ethylene glycol could not be established. Suicide attempts with ethylene glycol are extremely rare in Curaçao, but unintentional drinking occurs due to its sweet taste ${ }^{[5]}$.

\section{CONCLUSION}

These cases demonstrate that the diagnostic picture of toxic alcohol poisoning can be confusing despite in-hospital observation and that a priori opinions may be incorrect. Physicians often have to infer a diagnosis based on suspicion and initiate therapy before establishing a diagnosis as a delay may result in death.

\section{REFERENCES}

1. Boba A. Management of acute alcoholic intoxication. Am J Emerg Med 1999;17:431.

2. Barceloux DG, Bond GR, Krenzelok EP, Cooper H, Vale JA. American Academy of Clinical Toxicology practice guidelines on the treatment of methanol poisoning. Clin Toxicol 2002;40:415-446.

3. Brick J. Standardization of alcohol calculations in research. Alcohol Clin Exp Res 2006;30:1276-1287.

4. Jones AW. Evidence-based survey of the elimination rates of ethanol from blood with applications in forensic casework. Forensic Sci Int 2010;200:1-20.

Berend K, Duits AJ. The role of the clinical laboratory in diagnosing acid-base disorders. Crit Rev Clin Lab Sci 2019;56:147-169.

Halperin ML, Hammeke M, Josse RG, Jungas RL. Metabolic acidosis in the alcoholic: a pathophysiologic approach. Metabolism 1983;32:308-315.

Kraut JA, Mullins ME. Toxic alcohols. N Eng J Med 2018;378:270-280.

Yost DA. Acute care for alcohol intoxication. Postgrad Med 2009;112(6):14-26.

Chu J, Wang RY, Hill NS. Update in clinical toxicology. Am J Respir Crit Care Med 2002;166:9-15.

10. Jammalamadaka D, Raissi S. Ethylene glycol, methanol and isopropyl alcohol intoxication. Am J Med Sci 2010;339:276-281.

11. Kraut JA, Kurtz I. Toxic alcohol ingestions: clinical features, diagnosis, and management. Am Soc Nephrol 2008;3:208-225.

12. Kostic MA, Dart RC. Rethinking the toxic methanol level. Clin Toxicol 2003;41:793-800. 